PROCEEDINGS OF THE

AMERICAN MATHEMATICAL SOCIETY

Volume 139, Number 12, December 2011, Pages 4319-4329

S 0002-9939(2011)11217-6

Article electronically published on July 26, 2011

\title{
NONDEGENERACY OF THE SADDLE SOLUTION OF THE ALLEN-CAHN EQUATION
}

\author{
MICHAE KOWALCZYK AND YONG LIU
}

(Communicated by Yingfei Yi)

\begin{abstract}
A solution of the Allen-Cahn equation in the plane is called a saddle solution if its nodal set coincides with the coordinate axes. Such solutions are known to exist for a large class of nonlinearities. In this paper we consider the linear operator obtained by linearizing the Allen-Cahn equation around the saddle solution. Our result states that there are no nontrivial, decaying elements in the kernel of this operator. In other words, the saddle solution of the Allen-Cahn equation is nondegenerate.
\end{abstract}

\section{INTRODUCTION}

In this paper we consider the Allen-Cahn equation:

$$
\Delta u+f(u)=0, \quad \text { in } \mathbb{R}^{2} .
$$

The nonlinear function $f:[-1,1] \rightarrow \mathbb{R}$ is assumed to be smooth (at least $\mathcal{C}^{2}$ ), odd, and in addition such that the following holds:

$$
\begin{aligned}
& f( \pm 1)=f(0)=0, \quad f^{\prime}( \pm 1)<0, \\
& u \mapsto \frac{f(u)}{u} \quad \text { is strictly decreasing on }(0,1) .
\end{aligned}
$$

A function $U: \mathbb{R}^{2} \rightarrow(-1,1)$ is called a saddle solution of the problem (1.1) if $U(x, y)$ has the same sign as $x y$. It is proven in [6] that there exists a unique saddle solution of (1.1). Notice that by definition $U(0, y)=0=U(x, 0)$. Moreover, it is shown that $U$ is odd with respect to the $x$ and $y$ axes. Finally, a very important property of the saddle solution is that $U_{x}>0$ when $y>0$, and $U_{x}<0$ when $y<0$.

The problem of existence and qualitative properties of saddle type solutions has received a considerable amount of attention in recent years. In particular, in all even dimensions, saddle type solutions were found in 4. These solutions vanish along the Simons cone. Obviously each of these solutions can be extended trivially to odd dimensions, thus giving another example of a saddle type solution. In addition, it is not hard to show that, for example in dimension $n=3$, there exists a saddle-type

Received by the editors September 17, 2010.

2010 Mathematics Subject Classification. Primary 35B08, 35P99, 35Q80.

Key words and phrases. Allen-Cahn equation, nondegeneracy.

The first author was partially supported by Chilean research grants Fondecyt 1090103, Fondo Basal CMM-Chile, and Project Añillo ACT-125 CAPDE.

The second author was partially supported by Chilean research grants Fondecyt 3100011 and Fondo Basal CMM-Chile and doctoral grants of North China Electric Power University.

(C)2011 American Mathematical Society Reverts to public domain 28 years from publication 
solution vanishing along the three coordinate planes. Finally, in dimension $n=2$, solutions whose zero level set has the symmetry of a regular $2 k$-polygon and consists of $k$ straight lines passing through the origin were found in [1.

In this paper we will consider the linearization of the equation (1.1) around the saddle solution, i.e., the operator $L$ defined by

$$
L h \equiv \Delta h+f^{\prime}(U) h .
$$

Let us observe that functions $U_{x}, U_{y}$ satisfy

$$
L U_{x}=L U_{y}=0 .
$$

Geometrically these functions correspond to the invariance of the problem (1.1) with respect to translations in the direction of the coordinate axes. Strictly speaking, they do not belong to the kernel of the operator $L$, understood as a subset of $L^{2}\left(\mathbb{R}^{2}\right)$, and, because of their geometric interpretation, we refer to them as the Jacobi fields ("along" $U$ ). Observe that, since (1.1) is invariant with respect to rotations, there is another Jacobi field: $\nabla U \cdot(-y, x)=-y U_{x}+x U_{y}$. Note that $U_{x}, U_{y}$ are bounded functions, and $\nabla U \cdot(-y, x)$ is an unbounded function. While the knowledge of all Jacobi fields is in principle not necessary to develop a suitable invertibility theory of the linearized operator $L$, the classification of the decaying Jacobi fields is crucial for such a theory. In this paper we prove the following:

Theorem 1.1. Assume that $h$ is a solution of the problem $L h=0$ such that $\lim _{z \rightarrow \infty} h(z)=0$. Then $h \equiv 0$.

Observe that if we consider $L$ to be an operator in $L^{2}\left(\mathbb{R}^{2}\right)$ with $D(L)=H^{2}\left(\mathbb{R}^{2}\right)$, then in particular our theorem implies that $\operatorname{Ker}(L)=\{0\}$. Another way to state this is to say that the saddle solution is nondegenerate.

To explain the importance of our result in the theory of the Allen-Cahn equation in low dimensions, we observe that the saddle solution $U$ is a special case of the so-called multiple-end solution. To explain this concept we introduce the unique, odd and increasing heteroclinic solution $H$ of

$$
H^{\prime \prime}+f(H)=0, \quad H( \pm \infty)= \pm 1 .
$$

Letting for example $u(x, y)=H(x)$ we obtain a 2-end solution of (1.1). In general $u$ is a $k$-end solution to (1.1) if, outside a compact set containing the origin, the zero level set of $u$ consists of $k$ curves $\gamma_{j}, j=1, \ldots, k$, each of which is asymptotically a straight line. Moreover, along $\gamma_{j}$ and far from the origin, we have $u(x, y) \sim$ $\pm H$ (dist $\left.\left((x, y), \gamma_{j}\right)\right)$, where dist denotes a choice of the signed distance function. A precise definition of the notion of the multiple-end solution was introduced in 8 . In the same paper it has been proven that a multiple-end solution with any even number of nearly parallel ends always exists. This means in particular that a 4-end solution whose ends are asymptotically almost parallel exists. On the other hand, the saddle solution is another example of a 4-end solution, but this time with ends which are orthogonal to each other. It is natural to conjecture that these two types of solutions belong to the same connected component of the solution set of (1.1). Indeed, under the assumption that the saddle solution is nondegenerate, it is shown in [7] that there are multiple-end solutions "nearby". The angles between the ends of these solutions are close to $\pi / 2$. In this sense Theorem 1.1 can be seen as a first important step towards proving the conjecture mentioned above. We remark that this conjecture, which concerns the classification of all the 4-end solutions, can be 
looked at as an analogous version of the well-known result in the theory of minimal surfaces, which roughly speaking states that any of the singly periodic minimal surfaces in $\mathbb{R}^{3}$ that have four Scherk type ends belongs to the second Scherk surface family; for more details, see for example [13] and the references therein.

Let us now comment about the role played by the hypothesis (1.2) in the question of existence of the saddle solution of (1.1). Gui (see [11] and the references therein) showed, among other things, the existence of the unique saddle solution without assuming that $f(u) / u$ is strictly decreasing in $(0,1)$. We believe that the saddle solution is still nondegenerate in this case. One of the questions addressed in [1] is that of the necessary and sufficient condition for the existence of the saddle solution. While (1.2) and the oddness of $f$ (without the monotonicity of $f(u) / u$ ) is a sufficient condition for the existence, it is not known whether it is also a necessary condition. However it is shown in [11] that if a saddle solution exists, then

$$
\int_{-1}^{0} \sqrt{F(t)} d t=\int_{0}^{1} \sqrt{F(t)} d t, \quad \text { where } F^{\prime}(t)=-f(t) .
$$

This suggests that when $f$ is not an odd but just a bistable, balanced nonlinearity, rather then speaking of saddle solutions one should consider multiple-end solutions with ends that are asymptotically orthogonal, or nearly orthogonal. While proving an existence result for such solutions, with an approach mimicking that of [6] or [1], is in principle possible, it seems, at the same time, to be rather challenging. This is because in general (i.e., without the symmetry) we have no a priori information about the zero level set of the solution sought. On the other hand, it is quite plausible that for a nonlinearity which is a small perturbation of an odd function the existence result can be proven using the implicit function theorem. In the implementation of this program, the nondegeneracy of the saddle solution should be crucial.

\section{Preliminary Results}

We will first collect a few well-known results about some special solutions of the Allen-Cahn equation.

By $H$ we will always denote the unique, increasing heteroclinic solution of the Allen-Cahn equation in one dimension introduced in (1.4). Setting $\beta=\sqrt{\left|f^{\prime}(1)\right|}$ we have the following asymptotic formulas:

$$
H(t) \sim 1-A e^{-\beta t}, \quad H^{\prime}(t) \sim \beta A e^{-\beta t}, \text { as } t \rightarrow+\infty,
$$

where $A>0$ is a constant. Similar formulas hold when $t \rightarrow-\infty$. Note also that $H^{\prime}(t)>0, t \in \mathbb{R}$.

The heteroclinic solution is nondegenerate in the sense that the only bounded solution of

$$
\mathbb{L} \phi=0, \quad \text { where } \mathbb{L} \phi=\phi^{\prime \prime}+f^{\prime}(H) \phi,
$$

is the derivative of the heteroclinic solution $H$, i.e., $\phi=H^{\prime}$. More generally, if we consider the eigenvalue problem

$$
\mathbb{L} \phi=-\lambda \phi,
$$

then this problem has a finite, or infinite, increasing sequence of eigenvalues $\lambda_{0}=$ $0<\lambda_{1} \cdots \leq \beta^{2}$, where $\beta=\sqrt{\left|f^{\prime}(1)\right|}$. 
Considering the saddle solution $U(x, y)$, the following is proven (Theorem 2.8 in [14]): for each $\mu<\sqrt{\lambda_{1}}$, there exists $C_{\mu}>0$ such that

$$
|U(x, y)-H(x)| \leq C_{\mu} e^{-\mu|y|} .
$$

As a corollary of this (Corollary 2.9 in [14) one gets a similar estimate for the derivatives of the function $v(x, y):=U(x, y)-H(x)$ :

$$
\left|\partial^{\alpha} v(x, y)\right| \leq C_{\mu} e^{-\mu|y|},
$$

where $\alpha$ is a multi-index such that $|\alpha| \leq 3$. Clearly, because of the symmetry of $U$, and with obvious modifications, the same is true with $H(x)$ replaced by $H(y)$.

Let us now summarize some known facts about the spectrum of the operator $-L$ introduced in (1.3). The existence of the principal eigenvalue $\Lambda_{1}$ and eigenfunction $\Phi_{1}$, which is simple, is standard. This eigenvalue can be characterized as the minimum of the Raleigh quotient:

$$
\Lambda_{1}=\inf _{\phi \in H^{1}\left(\mathbb{R}^{2}\right)} \mathcal{A}(\phi), \quad \mathcal{A}(\phi)=\frac{\int_{\mathbb{R}^{2}}|\nabla \phi|^{2}-f^{\prime}(U) \phi^{2}}{\|\phi\|_{L^{2}\left(\mathbb{R}^{2}\right)}^{2}} .
$$

In addition, in [14], a suitable test function, for which $\mathcal{A}$ becomes strictly negative, is constructed, thus showing that $\Lambda_{1}<0$. Furthermore, see for example [14, it is not hard to see that the essential spectrum of $-L$ is the half line $[0, \infty)$. Finally we observe that a natural conjecture is that in fact $\Lambda_{1}$ is the only negative eigenvalue in the spectrum of $-L$ (i.e. $U$ has Morse index 1). Under the assumption that $f(u)=2 u-2 u^{3}$, a computer assisted proof of this fact is given in 14. In this respect, we would like to point out that the saddle-type solutions vanishing along Simons' cone in dimensions $n=4,6$ were found to have infinite Morse index (4, [3]). On the other hand, nondegeneracy of the saddle solution should imply that its Morse index is finite for any of the nonlinearity satisfying (1.2). In this context it is worth mentioning that there are related results in the theory of minimal surfaces; see 9 .

The above statements will be used later on, sometimes without any special reference.

We will now proceed to prove some preliminary facts about the operator $L$. To begin with we show that any bounded Jacobi field is a linear combination of $U_{x}, U_{y}$ and another Jacobi field with even symmetry.

Lemma 2.1. Suppose that $h$ is a bounded, smooth solution of $L h=0$. Then there exist constants $a, b$ such that $\varphi \equiv h-a U_{x}-b U_{y}$ is an even function of $(x, y)$, i.e., $\varphi(x, y)=\varphi(-x, y)=\varphi(x,-y)$.

Proof. We first decompose $h$ into the even and odd functions of $y$ :

$$
h(x, y)=\frac{1}{2}(h(x, y)+h(x,-y))+\frac{1}{2}(h(x, y)-h(x,-y)),
$$

and denote the odd part $\frac{1}{2}(h(x, y)-h(x,-y))$ by $\phi$ for simplicity. Then, obviously, $\phi(x, 0)=0$. Recall that $U_{x}(x, 0)=0$ and $U_{x}(x, y)>0$ for $y>0$.

We would like to adapt the method of [2], [10] to prove that

$$
\phi=a U_{x}
$$

for some constant $a$. 
Obviously, from the equalities

$$
\Delta U_{x}+f^{\prime}(U) U_{x}=0
$$

and

$$
\Delta \phi+f^{\prime}(U) \phi=0,
$$

it follows that for any $\varepsilon>0$,

$$
\operatorname{div}\left(U_{x}^{2} \nabla\left(\frac{\phi}{U_{x}}\right)\right)=0, \quad|y|>\varepsilon
$$

Denote $g:=\frac{\phi}{U_{x}}$ and multiply both sides of (2.4) with $g \xi_{R}^{2}(r)$, where $\xi_{R}(r):=$ $\xi\left(\frac{r}{R}\right), r=\sqrt{x^{2}+y^{2}}$ and $\xi$ is a cutoff function satisfying

$$
\xi(s)= \begin{cases}1, & 0<s<1 \\ 0, & s>2\end{cases}
$$

Then we have

$$
\int_{|y|>\varepsilon} g \xi_{R}^{2} \operatorname{div}\left(U_{x}^{2} \nabla g\right) d x d y=0 .
$$

Using the divergence theorem, one gets

$$
\int_{|y|=\varepsilon} g \xi_{R}^{2} U_{x}^{2} \nabla g \cdot \nu d x-\int_{|y|>\varepsilon} U_{x}^{2}\left\{\xi_{R}^{2} \nabla g+g \nabla\left(\xi_{R}^{2}\right)\right\} \cdot \nabla g d x d y=0,
$$

where $\nu$ is the unit outward normal to the boundary of the region $\{(x, y) \in$ $\left.\mathbb{R}^{2}:|y|>\varepsilon\right\}$. Consequently, if we set

$$
\Omega:=\left\{(x, y) \in \mathbb{R}^{2}: R<r<2 R \text { and }|y|>\varepsilon\right\},
$$

then it follows that

$$
\begin{aligned}
\int_{|y|>\varepsilon} U_{x}^{2} \xi_{R}^{2}|\nabla g|^{2} d x d y= & -\int_{\Omega} \frac{2}{R} g U_{x}^{2} \xi_{R} \xi^{\prime}\left(\frac{r}{R}\right) \nabla r \cdot \nabla g d x d y \\
& +\int_{|y|=\varepsilon} g U_{x}^{2} \xi_{R}^{2} \nabla g \cdot \nu d x \\
\leq & \frac{2}{R}\left\{\int_{\Omega} g^{2} U_{x}^{2}\left|\xi^{\prime}\left(\frac{r}{R}\right)\right|^{2} d x d y\right\}^{\frac{1}{2}}\left\{\int_{\Omega} U_{x}^{2}\left|\xi_{R}\right|^{2}|\nabla g|^{2} d x d y\right\}^{\frac{1}{2}} \\
& +\int_{|y|=\varepsilon} g U_{x}^{2} \xi_{R}^{2} \nabla g \cdot \nu d x .
\end{aligned}
$$

For the last term of the above inequality, it is easily seen that

$$
\begin{aligned}
\int_{|y|=\varepsilon} g U_{x}^{2} \xi_{R}^{2} \nabla g \cdot \nu d x & =\int_{|y|=\varepsilon} g \xi_{R}^{2}\left(\nabla \phi U_{x}-\phi \nabla U_{x}\right) \cdot \nu d x \\
& =\left(\int_{y=-\varepsilon}-\int_{y=\varepsilon}\right) g \xi_{R}^{2}\left(\phi_{y} U_{x}-\phi U_{x y}\right) d x \\
& =2 \int_{y=-\varepsilon} \xi_{R}^{2}\left(\phi \phi_{y}-\frac{\phi^{2}}{U_{x}} U_{x y}\right) d x .
\end{aligned}
$$

To estimate this integral, we observe that since $U_{x}(x, y)>0$ for $y>0$ and $U_{x}(x, 0)=0$, then by the Hopf lemma, $U_{x y}(x, 0)>0$. Hence using the fact that $\phi(x, 0)=0$ and L'Hospital's rule, for any $x \in \mathbb{R}$, we obtain

$$
\lim _{y \rightarrow 0} \frac{\phi^{2}(x, y)}{U_{x}(x, y)} U_{x y}(x, y)=0 .
$$


This combined with (2.6) then yields that for fixed $R$,

$$
\int_{|y|=\varepsilon} g U_{x}^{2} \xi_{R}^{2} \nabla g \cdot \nu d x \rightarrow 0, \text { as } \varepsilon \rightarrow 0 .
$$

Letting $\varepsilon \rightarrow 0$ in (2.5), using the boundedness of $\phi$, we can deduce

$$
\int_{\mathbb{R}^{2}} U_{x}^{2} \xi_{R}^{2}|\nabla g|^{2} d x d y \leq C\left\{\int_{\{R<r<2 R\}} U_{x}^{2}\left|\xi_{R}\right|^{2}|\nabla g|^{2} d x d y\right\}^{\frac{1}{2}},
$$

which then leads to

$$
\int_{\mathbb{R}^{2}} U_{x}^{2} \xi_{R}^{2}|\nabla g|^{2} d x d y \leq C
$$

Now we shall let $R \rightarrow+\infty$ in (2.7) and find that

$$
\int_{\mathbb{R}^{2}} U_{x}^{2}|\nabla g|^{2} d x d y=0,
$$

which implies that for some constant $a$,

$$
\phi=a U_{x} .
$$

Having proved such an identity for the function $\phi$, we now further decompose $\frac{1}{2}(h(x, y)+h(x,-y))$ into the odd and even functions of $x$ :

$$
\begin{aligned}
\frac{1}{2}(h(x, y)+h(x,-y))= & \frac{1}{4}[(h(x, y)+h(x,-y))-(h(-x, y)+h(-x,-y))] \\
& +\frac{1}{4}[(h(x, y)+h(x,-y))+(h(-x, y)+h(-x,-y))] .
\end{aligned}
$$

Applying similar arguments as above, we infer that

$$
h=a U_{x}+b U_{y}+\varphi
$$

for some constants $a, b$ and bounded function

$$
\varphi=\frac{1}{4}[(h(x, y)+h(x,-y))+(h(-x, y)+h(-x,-y))],
$$

which is even with respect to both the $x$ and $y$ variables and solves

$$
\Delta \varphi+f^{\prime}(U) \varphi=0 .
$$

Next, we wish to construct a positive supersolution of (1.3) outside a large ball. We will seek a supersolution of the form $P(x, y)=a(x) H^{\prime}(y)+a(y) H^{\prime}(x)$, where $a: \mathbb{R} \rightarrow \mathbb{R}_{+}$is a positive, smooth function defined by

$$
a(t)=1-\eta(t) e^{-\sigma|t|},
$$

and $\eta: \mathbb{R} \rightarrow[0,1]$ is a smooth cutoff function such that $\eta(t)=0,|t| \leq \frac{1}{2}$ and $\eta(t)=1,|t|>1$, and $\sigma \in\left(0, \sqrt{\lambda_{1}}\right)$ is fixed.

Lemma 2.2. There exists $r_{0}>0$ such that $L P \leq 0$ in $\mathbb{R}^{2} \backslash B_{r_{0}}$; in other words, $P$ is a positive supersolution of (1.3) outside a large ball centered at the origin.

Proof. We first calculate that

$$
\begin{aligned}
L P= & \left\{a^{\prime \prime}(x)-a(x)\left[f^{\prime}(H(y))-f^{\prime}(U)\right]\right\} H^{\prime}(y) \\
& +\left\{a^{\prime \prime}(y)-a(y)\left[f^{\prime}(H(x))-f^{\prime}(U)\right]\right\} H^{\prime}(x) .
\end{aligned}
$$


Now estimate (2.1) can be used with $\mu \in\left(\sigma, \lambda_{1}\right)$ to get

$$
\left|f^{\prime}(H(x))-f^{\prime}(U(x, y))\right| \leq M|U(x, y)-H(x)| \leq C_{\mu} e^{-\mu|y|}, \quad M=\sup _{-1 \leq t \leq 1}\left|f^{\prime \prime}(t)\right| .
$$

A similar estimate holds for $f^{\prime}(H(y))-f^{\prime}(U(x, y))$.

Fix such a $\mu \in\left(\sigma, \sqrt{\lambda_{1}}\right)$. For $|y|>1$, we have

$$
\begin{aligned}
a^{\prime \prime}(y)-a(y)\left[f^{\prime}(H(x))-f^{\prime}(U)\right] & =\left(1-e^{-\sigma|y|}\right)^{\prime \prime}-\left(1-e^{-\sigma|y|}\right)\left[f^{\prime}(H(x))-f^{\prime}(U)\right] \\
& =-\sigma^{2} e^{-\sigma|y|}-\left(1-e^{-\sigma|y|}\right)\left[f^{\prime}(H(x))-f^{\prime}(U)\right] \\
& \leq-\sigma^{2} e^{-\sigma|y|}+C_{\mu} e^{-\mu|y|}
\end{aligned}
$$

Hence there exists a constant $c_{1}>1$ such that if $|y|>c_{1}$, then

$$
a^{\prime \prime}(y)-a(y)\left[f^{\prime}(H(x))-f^{\prime}(U)\right]<0 .
$$

By symmetry, when $|x|>c_{1}$, we also have

$$
a^{\prime \prime}(x)-a(x)\left[f^{\prime}(H(y))-f^{\prime}(U)\right]<0 .
$$

Now using the fact that $H^{\prime}(x)>0, H^{\prime}(y)>0$ we then easily deduce that

$$
L P(x, y)<0, \quad \min \{|x|,|y|\}>c_{1} .
$$

On the other hand, for $|y| \leq c_{1}$, since $H^{\prime}(y) \geq C e^{-\beta|y|}$ we see immediately that

$$
\frac{a^{\prime \prime}(y)-a(y)\left[f^{\prime}(H(x))-f^{\prime}(U)\right]}{H^{\prime}(y)} \leq C
$$

and thus obtain

$$
\left|\frac{H^{\prime}(x)\left\{a^{\prime \prime}(y)-a(y)\left[f^{\prime}(H(x))-f^{\prime}(U)\right]\right\}}{H^{\prime}(y)}\right| \leq C e^{-\beta|x|} .
$$

Now it follows from this and (2.9) that when $|y|<c_{1}$ and at the same time $|x|>c_{1}$,

$$
\frac{\Delta P+f^{\prime}(U) P}{H^{\prime}(y)} \leq-\sigma^{2} e^{-\sigma|x|}+C_{\mu} e^{-\mu|x|}+C e^{-\beta|x|} .
$$

Since $\sigma<\min \{\mu, \beta\}$, we can find $c_{2} \geq c_{1}$ such that

$$
\Delta P+f^{\prime}(U) P \leq 0, \quad|x|>c_{2}, \quad|y|<c_{1} .
$$

Choosing $r_{0}>2 c_{2}$, by (2.10), (2.14), and the symmetry of $P$ we finally get the assertion of the lemma.

\section{Proof of the Theorem}

Now we are ready to prove Theorem 1.1 .

Proof. We assume to the contrary that $h \neq 0$. If $h$ does not change sign, then the proof is in fact simpler and the reader can go directly to Step 2 of the proof. If on the other hand $h$ changes sign, then we need: 
Step 1. Using Lemma 2.1 and the fact that $U_{x}, U_{y} \notin L^{2}\left(\mathbb{R}^{2}\right)$ we infer that $h$ is even, i.e., that it satisfies

$$
h(x, y)=h(-x, y)=h(x,-y) .
$$

Consider any connected component $\mathfrak{C}$ of its zero level set $\left\{z \in \mathbb{R}^{2}: h(z)=0\right\}$. We observe that in general $\mathfrak{C}$ is an immersed curve (see [5] or [12]). Another observation we make is that in any compact set the number of self-intersections of $\mathfrak{C}$ must be finite; this is because from Corollary 1 in 12 it follows that the common zeros of $h$ and $\nabla h$ cannot accumulate.

We first show that $\mathfrak{C}$ is bounded. To prove this, we will consider two cases.

Case 1. $\mathfrak{C} \cap\left\{(x, y) \in \mathbb{R}^{2}: x=0\right.$ or $\left.y=0\right\}=\emptyset$. In this case, $\mathfrak{C}$ is contained in one quadrant. Without loss of generality we may assume that $\mathfrak{C}$ is contained in the first quadrant. In the following, we show that in fact this cannot happen.

We start by observing that in this case, $\mathfrak{C}$ cannot contain any closed curve (here a closed curve means a homeomorphic image of the unit circle). Indeed, using that $U_{y}$ is a positive supersolution in the first quadrant we would get that, in the domain enclosed by this closed curve, $h=0$, which in turn would imply $h \equiv 0$ in $\mathbb{R}^{2}$. Therefore, we assert that $\mathfrak{C}$ is unbounded, since otherwise due to the local behavior of $h, \mathfrak{C}$ must contain a closed curve.

Now, we can find a continuous map $\eta:(0,1) \rightarrow \mathbb{R}^{2}$ such that $\eta(t) \in \mathfrak{C}$ for $t \in(0,1)$ and $|\eta(t)| \rightarrow+\infty$ as $t \rightarrow 0$ or 1 , and moreover $\eta$ does not have selfintersections. For $R$ large enough, we can find two numbers $a_{R}, b_{R}, 0<a_{R}<\frac{1}{2}<$ $b_{R}<1$, and a segment $l_{R}$ of the circular arc $\left\{(x, y) \in \mathbb{R}^{2}: x^{2}+y^{2}=R, x>0, y>0\right\}$ such that $\eta\left(\left[a_{R}, b_{R}\right]\right)$ and $l_{R}$ form a closed curve, which encloses a bounded domain $D_{R}$ in the first quadrant. Choose a connected component $D_{R}^{\prime}$ of the open set $\left\{z \in D_{R}: h(z) \neq 0\right\}$ such that $\eta\left(\frac{1}{2}\right) \in \partial D_{R}^{\prime}$. Without loss of generality we can assume that $h>0$ in $D_{R}^{\prime}$. It is easily seen that

$$
\int_{\partial D_{R}^{\prime}}\left(h \nabla U_{y} \cdot \nu-U_{y} \nabla h \cdot \nu\right) d s=\int_{D_{R}^{\prime}} \operatorname{div}\left(h \nabla U_{y}-U_{y} \nabla h\right) d x d y=0,
$$

where $\nu$ is the unit outward normal to the boundary of $D_{R}^{\prime}$. Notice that

$$
h(z)=0, \quad z \in \partial D_{R}^{\prime} \backslash\left(\partial D_{R}^{\prime} \cap l_{R}\right) .
$$

We claim that $\nabla h \cdot \nu<0$ on the smooth part of $z \in \partial D_{R}^{\prime} \backslash\left(\partial D_{R}^{\prime} \cap l_{R}\right)$. Indeed, this follows from the Hopf lemma since $h>0$ in $D_{R}^{\prime}$. Therefore, using (3.2), we find that for some positive number $c_{0}$ independent of $R$,

$$
\begin{aligned}
\left|\int_{\partial D_{R}^{\prime} \cap l_{R}}\left(h \nabla U_{y} \cdot \nu-U_{y} \nabla h \cdot \nu\right) d s\right| & =\left|\int_{\partial D_{R}^{\prime} \backslash \partial D_{R}^{\prime} \cap l_{R}}\left(h \nabla U_{y} \cdot \nu-U_{y} \nabla h \cdot \nu\right) d s\right| \\
& =\left|\int_{\partial D_{R}^{\prime} \backslash \partial D_{R}^{\prime} \cap l_{R}} U_{y} \nabla h \cdot \nu d s\right|>c_{0} .
\end{aligned}
$$


On the other hand, using the fact that $\left|\nabla U_{y}\right| \leq C_{\mu} e^{-\mu|y|}$ (see (2.2)),

$$
\begin{aligned}
\int_{\partial D_{R}^{\prime} \cap l_{R}}\left|h \nabla U_{y} \cdot \nu\right| d s & \leq \max _{z \in \partial D_{R}^{\prime} \cap l_{R}}|h(z)| \int_{0}^{\frac{\pi}{2}} C_{\mu} e^{-R \mu \sin \theta} R d \theta \\
& \leq C \max _{z \in \partial D_{R}^{\prime} \cap l_{R}}|h(z)| \\
& \rightarrow 0, \text { as } R \rightarrow+\infty .
\end{aligned}
$$

Similarly, using $\left|U_{y}\right| \leq C_{\mu} e^{-\mu|y|}$ and $\lim _{z \rightarrow \infty}|\nabla h(z)|=0$ (which follows from the elliptic estimates), we get

$$
\begin{aligned}
\int_{\partial D_{R}^{\prime} \cap l_{R}}\left|U_{y} \nabla h \cdot \nu\right| d s & \leq C \max _{z \in \partial D_{R}^{\prime} \cap l_{R}}|\nabla h(z)| \\
& \rightarrow 0, \text { as } R \rightarrow+\infty .
\end{aligned}
$$

Now, (3.3) with (3.4) and (3.5) give us a contradiction. Therefore Case 1 cannot happen.

Case 2. We assume that the set $\mathfrak{C}$ intersects with at least one of the coordinate axes, i.e., $\mathfrak{C} \cap\{y=0\} \neq \emptyset$ or $\mathfrak{C} \cap\{x=0\} \neq \emptyset$. Without loss of generality, we suppose that $\mathfrak{C} \cap\{y=0, x \geq 0\} \neq \emptyset$.

In this case, we consider the set $\mathfrak{C}^{\prime \prime}:=\mathfrak{C} \cap\{x \geq 0\}$. Since $h$ is even with respect to both the $x$ and $y$ axes, $\mathfrak{C}^{\prime}$ is connected and symmetric with respect to the $x$ axis.

We now claim that the set $\mathfrak{C}^{\prime \prime} \cap\left\{(x, y) \in \mathbb{R}^{2}: y=0\right\}$ consists of exactly one point. In fact, if there were two points, say $\left(x_{1}, 0\right)$ and $\left(x_{2}, 0\right), x_{1}<x_{2}$, belonging to this set, then for $x_{1}<t<x_{2}$, the point $(t, 0) \in \mathfrak{C}^{\prime}$. Otherwise, since $\mathfrak{C}^{\prime \prime}$ is connected and $h$ is even with respect to the $x$ axis, we could find a closed curve in $\mathfrak{C}^{\prime \prime}$. But this, by the argument already considered (Case 1), cannot happen. Therefore, by the evenness of $h$,

$$
\phi_{x}(t, 0)=\phi_{y}(t, 0)=0, \quad x_{1}<t<x_{2} .
$$

But (3.6) will contradict with the Hopf lemma. The claim then follows. The unique point of intersection of $\mathfrak{C}^{\prime}$ with the $x$ axis will be denoted by $p_{0}$.

To prove that $\mathfrak{C}$ is bounded we now argue by contradiction. Assuming that $\mathfrak{C}$ is unbounded, we infer that the set

$$
\mathfrak{C}^{\prime} \cap\left\{(x, y) \in \mathbb{R}^{2}: y \geq 0\right\}
$$

is unbounded due to the symmetry of $h$. We can then find a continuous map

$$
\theta=\left(\theta_{1}, \theta_{2}\right):[0,1) \rightarrow \mathbb{R}^{2}
$$

such that $\theta(0)=p_{0}, \theta(t) \in \mathfrak{C}^{\prime \prime} \cap\left\{(x, y) \in \mathbb{R}^{2}: y>0\right\}$ for $t \in(0,1)$ and $|\theta(t)| \rightarrow+\infty$ as $t \rightarrow 1$. Again we observe that the image of $\theta$ does not have self-intersections. Reflecting $\theta$ across the $x$ axis we get a continuous curve

$$
\tilde{\theta}=\left(\tilde{\theta}_{1}, \tilde{\theta}_{2}\right):(-1,1) \rightarrow \mathbb{R}^{2}
$$

defined by

$$
\left(\tilde{\theta}_{1}(t), \tilde{\theta}_{2}(t)\right):=\left\{\begin{array}{l}
\left(\theta_{1}(-t),-\theta_{2}(-t)\right),-1<t \leq 0, \\
\left(\theta_{1}(t), \theta_{2}(t)\right), 0 \leq t<1 .
\end{array}\right.
$$

Using this curve and the segment of the circular arc $\left\{(x, y) \in \mathbb{R}^{2}: x^{2}+y^{2}=R\right.$, $x \geq 0\}, R$ large enough, and arguing as in Case 1, we will get a contradiction. 
By the above arguments, if $h$ changes sign, any connected component $\mathfrak{C}$ of its zero level set is bounded. Clearly, $\mathfrak{C}$ will intersect with both $x$ and $y$ axes. We now prove that this fact, together with the existence of the supersolution $P$ outside a large ball, guarantees that the zero level set cannot have a sequence of connected components $\mathfrak{C}_{n}$ such that

$$
\inf _{(x, y) \in \mathfrak{C}_{n}}(|x|+|y|) \rightarrow+\infty .
$$

In fact, if there were two connected components $\mathfrak{C}_{1}, \mathfrak{C}_{2}$ such that $\mathfrak{C}_{1} \cup \mathfrak{C}_{2} \subset \mathbb{R}^{2} \backslash B_{r_{0}}$, where $r_{0}$ is as in Lemma 2.2. then, using in addition the symmetry of $h$, we can find two closed curves $l_{i} \subset \mathfrak{C}_{i}, i=1,2$, which both enclose the origin. Now using $P$ as a supersolution in the annulus-like region enclosed between $l_{1}$ and $l_{2}$, we get a contradiction.

In summary: using the local behavior of $h$, we infer that the zero level set of $h$ consists of finitely many connected components, all of which are bounded. This in turn means that the zero level set itself is bounded. Therefore, we can assume that $h(z)>0$ when $z$ is large enough, say $|z|>r_{1}$. This assumption is trivial when $h$ does not change sign.

Step 2. Define

$$
\omega(y):=\int_{-\infty}^{+\infty} h(x, y) H^{\prime}(x) d x, \quad y>r_{1} .
$$

By the choice of $r_{1}$ above we have that $\omega>0$, and since $\lim _{z \rightarrow \infty} h(z)=0$ we have $\lim _{y \rightarrow+\infty} \omega(y)=0$. Now we calculate:

$$
\begin{aligned}
\omega^{\prime \prime}(y) & =\int_{-\infty}^{+\infty} h_{y y}(x, y) H^{\prime}(x) d x \\
& =\int_{-\infty}^{+\infty}\left[-h_{x x}-f^{\prime}(U) h\right] H^{\prime}(x) d x \\
& =-\int_{-\infty}^{+\infty} h H^{\prime \prime \prime}(x) d x-\int_{-\infty}^{+\infty} f^{\prime}(U) h H^{\prime}(x) d x \\
& =\int_{-\infty}^{+\infty}\left[f^{\prime}(H(x))-f^{\prime}(U)\right] h H^{\prime}(x) d x .
\end{aligned}
$$

Applying the estimate (2.9) then results in

$$
\omega^{\prime \prime}-C_{\mu} e^{-\mu y} \omega \leq 0, \quad y>r_{1} .
$$

In particular $\omega$ is a positive supersolution of

$$
\rho^{\prime \prime}-C_{\mu} e^{-\mu y} \rho=0, \quad y>r_{1}, \quad \rho\left(r_{1}\right)=\frac{1}{2} \omega\left(r_{1}\right)>0 .
$$

Since $\rho \equiv 0$ is a subsolution, there should exist a positive, decaying solution $\rho^{*}$ of (3.8). However, a simple ODE analysis tells us that two linearly independent solutions of (3.8) behave like $\rho_{1}(y) \sim 1, \rho_{2}(y) \sim y$, as $y \rightarrow+\infty$. This implies that $\rho^{*} \equiv 0$, which is a contradiction and ends the proof of the theorem. 


\section{REFERENCES}

[1] F. Alessio, A. Calamai, and P. Montecchiari. Saddle-type solutions for a class of semilinear elliptic equations. Adv. Differential Equations, 12(4):361-380, 2007. MR2305872 (2007m:35051)

[2] H. Berestycki, L. Caffarelli, and L. Nirenberg. Further qualitative properties for elliptic equations in unbounded domains. Ann. Scuola Norm. Sup. Pisa Cl. Sci. (4), 25(1-2):69-94 (1998), 1997. Dedicated to Ennio De Giorgi. MR.1655510 (2000e:35053)

[3] X. Cabre and J. Terra. Qualitative properties of saddle-shaped solutions to bistable diffusion equations. Comm. Partial Differential Equations, 35(11):1923-1957 (2010). MR2754074

[4] X. Cabré and J. Terra. Saddle-shaped solutions of bistable diffusion equations in all of $\mathbb{R}^{2 m}$. J. Eur. Math. Soc. (JEMS), 11(4):819-843, 2009. MR2538506 (2010j:35175)

[5] E. N. Dancer. On the influence of domain shape on the existence of large solutions of some superlinear problems. Math. Ann., 285(4):647-669, 1989. MR1027764 (91h:35122)

[6] H. Dang, P. C. Fife, and L. A. Peletier. Saddle solutions of the bistable diffusion equation. Z. Angew. Math. Phys., 43(6):984-998, 1992. MR.1198672(94b:35041)

[7] M. del Pino, M. Kowalczyk, and F. Pacard. Moduli space theory for the Allen-Cahn equation in the plane. To appear in Trans. Amer. Math. Soc.

[8] M. del Pino, M. Kowalczyk, F. Pacard, and J. Wei. Multiple-end solutions to the Allen-Cahn equation in $\mathbb{R}^{2}$. J. Funct. Anal., 258(2):458-503, 2010. MR2557944(2010i:35133)

[9] D. Fischer-Colbrie. On complete minimal surfaces with finite Morse index in three-manifolds. Invent. Math., 82(1):121-132, 1985. MR808112 (87b:53090)

[10] N. Ghoussoub and C. Gui. On a conjecture of De Giorgi and some related problems. Math. Ann., 311(3):481-491, 1998. MR1637919 (99j:35049)

[11] C. Gui. Hamiltonian identities for elliptic partial differential equations. J. Funct. Anal., 254(4):904-933, 2008. MR22381198 (2009b:35112)

[12] P. Hartman and A. Wintner. On the local behavior of solutions of non-parabolic partial differential equations. Amer. J. Math., 75:449-476, 1953. MR0058082 (15:318b)

[13] J. Pérez and M. Traizet. The classification of singly periodic minimal surfaces with genus zero and Scherk-type ends. Trans. Amer. Math. Soc., 359(3):965-990 (electronic), 2007. MR:2262839 (2007m:53010)

[14] M. Schatzman. On the stability of the saddle solution of Allen-Cahn's equation. Proc. Roy. Soc. Edinburgh Sect. A, 125(6):1241-1275, 1995. MR1363002(96j:35085)

Departamento de Ingeniería Matemática and CMM (UMi 2807, CNRS), Universidad de Chile, Casilla 170 Correo 3, Santiago, Chile

E-mail address: kowalczy@dim.uchile.cl

Departamento de Ingeniería Matemática and CMM (UMi 2807, CNRS), Universidad de Chile, Casilla 170 Correo 3, Santiago, Chile

Current address: School of Mathematics and Physics, North China Electric Power University, Beijing, People's Republic of China 102206

E-mail address: yliu@dim.uchile.cl 